\title{
Earth Measurements Via Satellites $\left(^{*}\right)$
}

\author{
A. L. Cotchen \\ Ricevuto il 19 Febbraio 1963
}

SUMAARY. - Geodetic parameters and intercontinental ties can be determined using a new technique which combines electronic distancemeasuring equipment (DME) and artificial satellites.

In initial field tests, average range resolutions of $30 \mathrm{~cm}$ have been obtained at distances greater than $3000 \mathrm{~km}$.

Rissusto. - Nella nota l'A. mette in evidenza l'uso di nuove tecniche per determinare i parametri geodetici e i collegamenti intercontinentali, abbinando l'equipaggiamento elettronico per misura-distanze (I)ME) ai satelliti artificiali.

The purpose of this paper is to introduce the concept of using artificial earth satellites for measuring the size and shape of the earth, and the relative position of places on its surface. An advanced electronic system which has been built to implement this concept will be described in general terms, with a review of the experimental data obtained to date. This system, called Secor, provides a new and powerful tool for extending man's knowledge of the planet on which he lives.

Before discussing Secor itself, I should like to talk about the basic building blocks of which it is composed ... electronic IDME (DistanceMeasuring Equipment). DME operates on a very simple principle, which says that the phase shift experienced by any propagated electromagnetic wave is directly proportional to the propagation path length. Therefore, if this phase shift can be measured by comparing the wave at the beginning of the path with the wave at the end, the path length

(*) Nota presentata al $2^{\circ}$ Congresso Internazionale Tecnico Scientifico dello Spazio. Roma, 19.23 Giugno 1962. 
"an be determined. Making such a measurement if the beginning and end of the path are far apart poses serious problems, which may be easily avoided, however: the beginning and end are at the same place, but in traveling from one to the other, the wave is propagated via the point whose distance is of interest. Then this point's distance is, of course, exactly half the path length indicated by the phase difference measurement at the common beginning-end.

For example, a c-w signal is transmitted from a ground station to some airborne vehicle carrying a transponder; the transponder rereives the signal and retransmits it back to the ground station, where it is compared in phase with the original transmission to determine the distance from the ground station to the airborne vehicle. Techniques have been developed in recent years which make this kind of distance measurement, using phase comparison of continuous-wave signals, extremely accurate. Or, more precisely speaking, extremely aceurate whenever the phase difference measured is less than one wavelength $\left(360^{\circ}\right)$; phase differences greater than that raise measurement problems that are not readily solved. In general, the accuracy of measurement, expressed as a certain small fraction of a wavelength, depends only on the equipment used, but does not depend on the wavelength; in other words, equipment that can measure a phase difference as small as one thousandth of a wavelength can make path length measurements with a resolution of $1 \mathrm{~km}$ if the wavelength is $1000 \mathrm{~km}$, but with a resolution of 1 c $\mathrm{m}$ if the wavelength is 10 meters.

In summary, then, the two fundamental facts abont phase-comparison DME are (1) extreme accuracy inversely proportional to wavelength and (2) but only at distances less than one wavelength. Thus, we must rhoose a short wavelength (high frequency) to ensure high accuracy, and at the same time choose a long wavelength (low frequen(y) if we want to measure long distances. Here is the basic dilemma. It is solved, in Cubic DME, by temporarily ignoring the actual transmitted frequency (carrier) and considering modulation signals impressed upon it. These modulation signals undergo exactly the same sort of pathlength-dependent phase shift I previously described, and in Cubic DME there are usually four or five of them, covering the range between the longest distance ever to be measured (say, 20,000 km) and the desired aceuracy (say, 1 meter). The lower-frequency modulating signals provide the unambiguous data revealing less and less roughly how far away the transponder is, while the highest-frequency modulating signal pinpoints the distance, much as the numerals in a 4-digit number tell 
you, one by one from the left, the thousands, hundreds, decades, and finally the units involved.

So much for a simple DVIE, measuring even very long ranges with very ligh accuracies. By measuring the distance to some airborne object, it tells us in effect that the object is somewhere on a hemisphere whose center is at the ground station and whose madius is the measured distance. If the distance to the same airborne object is simultaneously measured from a second ground station somewhere else on the earth's surface, a second hemisphere is established, and the object must lie on this second hemisphere as well. Therefore, it must lie on the semicircle which is the intersection of the two hemispheres. A distance measurement from a third ground station provides a hemisphere which intersects the semicircle at only one point, thereby uniquely determining the location of the object in space.

Secor measures spatial positions in just this way, using three or more ground stations, except that the measurements are not made quite simultaneously. Instead, to permit using only a single transponder in the airborne vehicle, where space and weight are usually at a premium, this transponder is rapidly time-shared among the ground stations; system accuracy is not appreciably affected. Secor is being used today on major test ranges throughout the world for tracking and guiding both long-range and short-range missiles.

Now let's consider an actual earth measurement problem, like determining the distance between North America and Hawaii. Because of rertain difficulties associated with celestial observations, such distances are now known to within perhaps the nearest kilometer. With Secor, it is anticipated that this uncertainty can be reduced to as little as 30 meters. The fundamental technique is as follows: (1) place three DME ground stations at accurately surveyed points, a few hundred miles apart, in North America; (2) place a fourth DME ground station in Hawaii; (3) orbit an artificial satellite carying a Secor transponder; (4) determine the satellite's orbit (with respect to North America) prerisely from the North American ground stations; (5) determine the Hawaiian station's position (with respect to the orbit) from the satellite.

Perhaps the last step requires a brief explanation. Is it moves along its orbit, the satellite can measure the distance to the Hawaian station whenever the latter is "in sight", which might be for several thousand orbital miles. Measurements at merely three points, perhaps 500 miles apart, however, are enough to pinpoint the Hawaiian site; we have three intersecting hemispheres, just as before, except that now 
their centers lie along the orbit while their only point of intersection is on the earth's surface.

At present, the Secor earth-measurement program is preparing for large-scale evaluation. A number of helicopter-transportable ground stations have been built and tested against Secor transponders carried aboard three earth satellites; in these tests, average range resolutions of $30 \mathrm{~cm}$ were obtained at distances up to 2000 miles. More ground stations, of advanced and ruggedized design, as well as additional transponders and satellites, are built and building. It is anticipated that the first intercontinental clata will be available within less than a year, thus marking the begimning of a long and useful life for Secor as a unique scientific instrument for exploring the earth from the newest of its moons. 